
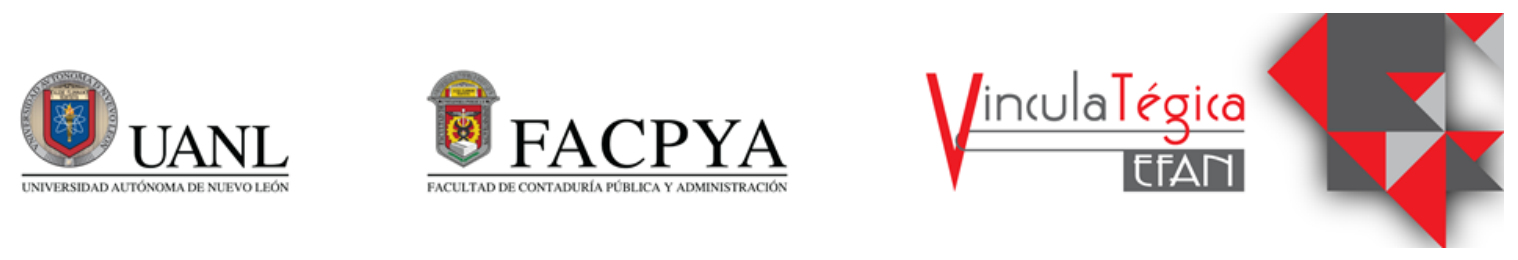

\title{
México: un país emergente con oportunidad económica sustentable; un ensayo desde una perspectiva de innovación y competitividad
}

\author{
Emmanuel Herrera Esquivel ${ }^{1}$, Jorge Treviño Montemayor $^{2}$ y Eduardo Cerecedo Cruz $^{3}$ \\ ${ }^{1}$ Instituto Rosario Castellanos, ing.e.herrera@hotmail.com, Ciudad de México, 5582522870 \\ ${ }^{2}$ Universidad Autónoma de Nuevo León, jgtrevinom@yahoo.com.mx, Ciudad Universitaria, 8183626199 \\ ${ }^{3}$ Instituto Rosario Castellanos, jefatura.contaduriayfinanzas.irc@gmail.com, Ciudad de México, 5512390160
}

Información del artículo revisado por pares

Fecha de aceptación: junio-2021

Fecha de publicación en línea: octubre-2021

DOI: https://doi.org/10.29105/vtga7.2-44

\begin{abstract}
Sustainable Development and renewable energy sources as generators of the knowledge economy in the reengineering of companies (SXXI Economic Transformation and Evolution). Today, we know that the concept of sustainable development has as a uniform and related objective between the economic and productive growth of society, as well as the conscious exploitation of natural resources. In this sense, all companies are designed to be aware of opportunities, and not weaknesses, that can be generated in the market, specifically in the redesign of the industry. It is estimated that the economy could double by the year 2042 if it grows (constantly, or close to) at $+-2.3 \%$ of its average annual rate between the years 2016 and 2050, taking into account that emerging markets and developing countries or growth of economies such as China, India, Brazil, Indonesia, Russia, Turkey and Mexico. Also according to the report, the great challenges for governments will be long-term sustainable growth policies (development plan), since the need to develop economic, legal and social political institutions could generate incentives for innovation, trust in finances, economies and stability for the generation of new businesses and investment confidence.
\end{abstract}

Keys Word: Economic development, Government policy and regulation, Environment and development

JEL: F63, G18, Q56

\section{Resumen}

El Desarrollo Sustentable y las fuentes de energía renovables como generadoras de la economía del conocimiento en la re-ingeniería de las empresas (Transformación y Evolución económica del SXXI). Hoy en día, sabemos que el concepto de desarrollo sustentable tiene como objetivo uniforme y relacionado entre el crecimiento económico y productivo de la sociedad, así como la explotación consciente de los recursos naturales. En este sentido, todas las empresas están diseñadas para estar alertas de las oportunidades, y no de las debilidades, que se pueden generar en el mercado, específicamente en el rediseño de la industria. Se estima que la economía podría duplicarse para el año 2042 si es que crece (constantemente, o cerca) al +-2.3\% de su tasa anual promedio entre los años de 2016 a 2050 tomando en cuenta que los mercados emergentes y los países en desarrollo o crecimiento de las economías como China, India, Brasil, Indonesia, Rusia, Turquía y México. También de acuerdo con el informe, los grandes desafíos de los gobiernos serán políticas de crecimiento sostenible a largo plazo (plan de desarrollo), ya que la necesidad de desarrollo instituciones políticas económicas, legales y sociales podrían generar incentivos sobre innovación, confianza en las finanzas, economías y estabilidad para la generación de nuevos negocios y confianza de inversión. 
Palabras Clave: Desarrollo económico, Política y regulación del gobierno, Medio ambiente y desarrollo

\section{INTRODUCCIÓN}

Hoy en día las economías emergentes apuntan a ser grandes desarrolladores renovables de sustitutos de hidrocarburos, mientras que los países desarrollados apuestan por apuntalar estrategias para combatir el cambio climático, desde la parte social, económica, industrial y gubernamental (joebiden.com, 2021; Oppenheimer, 2021), que si bien, parte del desarrollo sustentable debería ser un cambio social pacífico y gradual, por lo que la sociedad estaría inmerso en modificar su estilo y hábitos de vida (Ramírez, Sánchez \& García, 2004); teniendo en cuenta la Agenda 2030 que las Naciones Unidas (NU, 2015) han adoptado para el Desarrollo Sostenible para hacer frente a los efectos invernadero y calentamiento global.

A finales del siglo XX, nace el concepto de bioeconomía, también conocida como economía biobasada, que se encarga de la evolución del conocimiento de la vida para productos nuevos y agradables con el medio ambiente (Hodson de Jaramillo, 2018), las cuales son un área de oportunidad de crecimiento económico, cultural social, industrial y comercial afines de combinar los recursos ambientales e ideales políticas que fomenten crecimiento económico teniendo argumentaciones contradictorias entre las propias naciones (Jacobs, 1991), en el que el papel gubernamental debería incentivar la innovación sustentable y concordante a un plan de desarrollo; hacer rendir eficaz y eficientemente los recursos naturales renovables con el objetivo de consolidar un proyecto a futuro (como el concepto natural de economía) para propiciar mayor calidad de vida para la sociedad, disminuir la pobreza y acrecentar la economía basado en procesos de transformación de productos en el margen de innovación y desarrollo, incitando a promover prácticas de transformación o productoras y de consumo sustentable (Badii et al., 2007).

México, está marcado en repuntar su desarrollo tecnológico y acrecentar su economía a través de los propios recursos naturales por lo que es rico en éstos, mismos que permiten ampliar una cartera de condiciones de transformación de diversos productos con una efectiva mano de obra, la cual debería ser formulada desde una trinchera pacífica, leal, visionaria, proyectista y justa en condiciones laborales, sociales, educativas, industriales y comerciales; dejando a un lado los pensamientos conservatorios (de la segunda) revolución industrial del siglo IX (entendiendo que la primera se adjudica al siglo XVIII) y apostar por mejoras en condiciones tecnológicas de procesos industriales para enfocar y salvaguardar las relaciones tanto internas, como externas del país (Oropeza, 2013 y Barcelatta, 2012).

México asume el objetivo de reducir en un 50\% sus emisiones de GEI al 2050, en relación con las emitidas en el año 2000. México aspira así a contribuir a un posible escenario de estabilización de las concentraciones de GEI en la atmósfera, a un nivel no superior a 450 partes por millón de bióxido de carbono equivalente (CO2e, que es la concentración de CO2 que causa el mismo nivel de forzamiento radiactivo que un determinado tipo y concentración de gases de efecto invernadero (Carrillo Fernández, 2012).

"El escenario de mitigación de largo plazo prevé que en 2020 México logre una reducción del 21\% y en 2030 del $41 \%$, respecto a la línea base de crecimiento de emisiones [escenario tendencial]" (Carrillo Fernández, 2012).

\section{HIPÓTESIS}


Las políticas públicas con las que actualmente cuenta México sobre Desarrollo Sostenible en miras hacia la Agenda 2030 establecida por la Asamblea General de la ONU son efectivas para un crecimiento económico sustentable, ambiental y social de acuerdo con los recursos con los que actualmente cuenta el país.

\section{MARCO TEÓRICO}

\section{Principios básicos: fundamentos sobre primeras necesidades económicas y desarrollo sustentable}

El Desarrollo Sustentable y las fuentes de energía renovables como generadoras de la economía del conocimiento en la re-ingeniería para las empresas pueden verse como la Transformación y Evolución económica del SXXI. Hoy en día, sabemos que el concepto de desarrollo sustentable tiene como objetivo uniforme y relacionado entre el crecimiento económico y productivo de la sociedad, así como la explotación consciente de los recursos naturales sin comprometer y poner en riesgo alguno, la vida del planeta y de los seres vivos y sus ecosistemas.

"A la par de la expansión eléctrica, las presiones internacionales y nacionales han empujado la evaluación del impacto ambiental logrando mayor especificidad y profundidad de la información científica del área donde se construirá el proyecto" (González 2011, 46).

Lo anterior, puede llevarse a cabo mediante sistemas técnicos y metodológicos capaces de investigar continuamente nuevas y mejores soluciones de acuerdo con estándares de calidad. Es por ello, que se tienen tres tipos de factores o tipos de sustentabilidad: Estrategia de Sustentabilidad Social, Estrategia de Sustentabilidad de la Economía, y Estrategia de Sustentabilidad del Medio Ambiente.

Es importante identificar y valorar la aportación que realizan las personas que juegan un papel importante en la ética y la cultura de sostenibilidad, los cuales implementa nuevos programas de acción de desarrollo y capacitación sostenible. Hace ya algunos meses, los Emiratos Árabes Unidos, iniciaron una misión espacial, al enviar un cohete a marte con la finalidad de generar economía del conocimiento para que los jóvenes en un futuro generen valor agregado mediante la gran información que se pudiera recabar de la misión espacial obteniendo economía del conocimiento, por el enfoque mismo, una misión explícita en estimular a la ciudadanía joven del presente en conocimiento comprensible y manejable al adoptar toma de decisiones.

En este sentido, todas las empresas están diseñadas para estar alertas a las oportunidades, y no tanto así a sus debilidades generalmente expuestas por el mercado, específicamente en el rediseño de la industria acoplados a un sistema de gestión de calidad mediante información procesada, analizada como minería de datos o metadatos; por lo que existen fundamentos y factores específicos en este proceso: Transición, Innovación, Ganas de trascender, Disciplina y talento, Esperanza de éxito. Cuando las empresas se encuentran en ese inter, existe una hostilidad en los mercados versus la nobleza a las necesidades de los clientes y el consumo que permitirán a las empresas poder realizar e identificar el algoritmo correcto para transcender en la aplicación y uso de las energías renovables; ya que hoy en día, empresa que no evolucione hacia un enfoque de desarrollo sustentable, se estima que en un $90 \%$ será una empresa que no trascienda y estará destinada al fracaso.

De acuerdo a lo anterior, empresas como: Biofarmas, Biocombustibles, Bioalimentos, son ramas industriales que serán de éxito en un futuro próximo teniendo el conocimiento ligado con la disciplina y el saber que genera riqueza en todos los sentidos, y busca dentro de esa riqueza, generar valor agregado mediante la innovación en pequeños, diminutos y casi imperceptibles cambios dentro de una organización 
que producen cambios de alto impacto; de tal forma, que para que esto suceda, se requiere de una cultura de educación y capacitación continua, siendo portadores básicos del crecimiento de la economía del conocimiento, ya que incrementan el desarrollo de habilidades, técnicas y conocimiento mediante la experiencia residual y sostenible de la innovación social, económica y ambiental; capacitando a quienes son parte del cambio, de la transformación y evolución de las transformaciones amigables con el medio ambiente.

\section{Políticas sustentables: necesidades sobre innovación y tecnología de energías bioambientales como oportunidades para México}

De acuerdo con el informe del World in 2050 de PwC (2021): The long view: how will the global economic order change by 2050? (La visión a largo plazo: ¿cómo cambiará el orden de la economía mundial para el año 2050?), ha realizado diversas proyecciones de crecimientos potenciales del Producto Interno Bruto (PIB) para aquellos países del mundo que tienen mejores economías, las cuales representan cerca del $85 \%$ del PIB global, basadas principalmente en un modelo sobre crecimiento global a futuro.

Se estima que la economía podría duplicarse para el año 2042 si es que crece (constantemente, o cerca) al +-2.3\% de su tasa anual promedio entre los años de 2016 a 2050 tomando en cuenta que los mercados emergentes y los países en desarrollo o crecimiento de las economías como China, India, Brasil, Indonesia, Rusia, Turquía y México con los que se esperan que en promedio crezcan por ahí del 3,5\% anual durante los próximos 30 años, a comparación de los países avanzados como los que componen el G7 como: Estados Unidos de América (EUA), Alemania, Canadá, Francia, Japón, Italia y Reino Unido que se estima que crecerán a un $1.6 \%$.

Debemos tener en cuenta y conocer, para que una economía se pueda considerar como Emergente debe tener o cumplir con los escenarios siguientes: Tener riqueza de recursos y materias primas; industria potencializada; estabilidad política; inversión extranjera directa; crecimiento en la confianza del consumidor; buenas relaciones comerciales con los mercados globales (Gurría, 2011); Bajo el mismo enfoque del informe de $\mathrm{PwC}$, podemos interpretar la siguiente información:

Tabla 1. PIB en Paridad de Poder Adquisitivo (PPA)

\begin{tabular}{|c|c|c|c|c|c|c|}
\hline & \multicolumn{2}{|c|}{ En el año 2016} & \multicolumn{2}{|c|}{ Estimación 2030} & \multicolumn{2}{|c|}{ Estimación 2050} \\
\hline $\begin{array}{l}\text { Posició } \\
\text { n }\end{array}$ & País & $\begin{array}{l}\text { PIB- } \\
\text { PPA }\end{array}$ & País & $\begin{array}{l}\text { PIB- } \\
\text { PPA }\end{array}$ & País & $\begin{array}{l}\text { PIB- } \\
\text { PPA }\end{array}$ \\
\hline 1 & China & 21,269 & China & 38,008 & China & 58,499 \\
\hline 2 & EUA & 18,562 & EUA & 23,475 & India & 44,128 \\
\hline 3 & India & 8,721 & India & 19,511 & EUA & 34,102 \\
\hline 4 & Japón & 4,932 & Japón & 5,606 & Indonesia & 10,502 \\
\hline 5 & Alemania & 3,979 & Indonesia & 5,424 & Brasil & 7,540 \\
\hline 6 & Rusia & 3,745 & Rusia & 4,736 & Rusia & 7,131 \\
\hline 7 & Brasil & 3,135 & Alemania & 4,707 & México & 6,863 \\
\hline 8 & Indonesia & 3,028 & Brasil & 4,439 & Japón & 6,779 \\
\hline 9 & $\begin{array}{l}\text { Reino } \\
\text { Unido }\end{array}$ & 2,788 & México & 3,661 & Alemania & 6,138 \\
\hline 10 & Francia & 2,737 & Reino Unido & 3,638 & Reino Unido & 5,369 \\
\hline 11 & México & 2,307 & Francia & 3,377 & Turquía & 5,184 \\
\hline 12 & Italia & 2,221 & Turquía & 2,996 & Francia & 4,705 \\
\hline
\end{tabular}


Grafica 1. PIB en Paridad de Poder Adquisitivo 2016

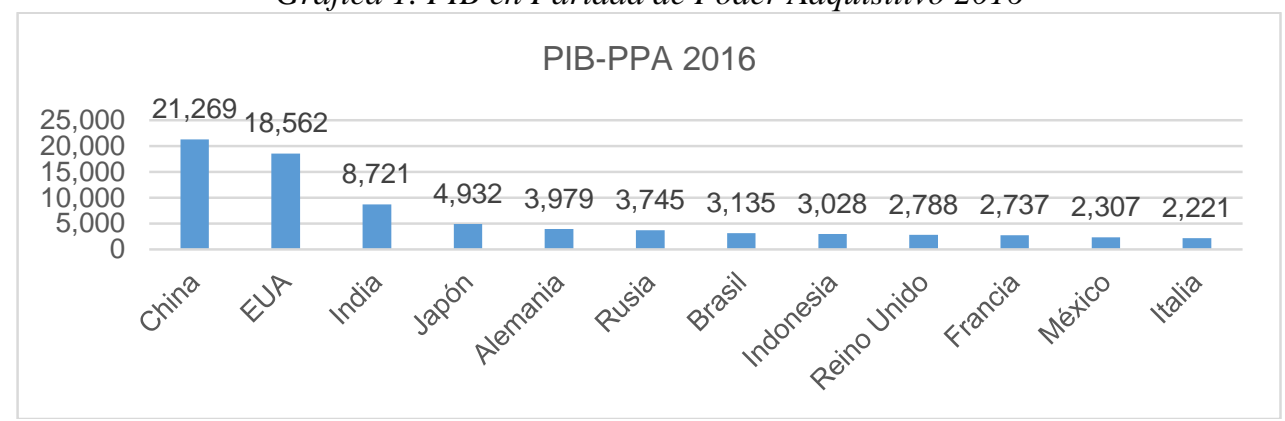

Fuente: PwC, 2021, Hacia el 2050: las economías emergentes tendrán el poder económico del mundo

Grafica 2. PIB en Paridad de Poder Adquisitivo 2030

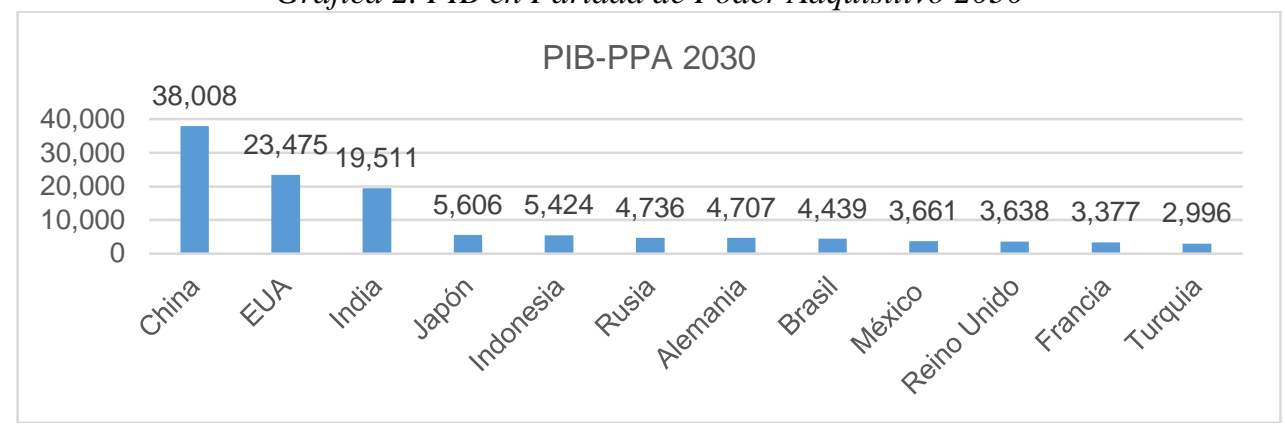

Fuente: PwC, 2021, Hacia el 2050: las economías emergentes tendrán el poder económico del mundo

Grafica 3. PIB en Paridad de Poder Adquisitivo 2050

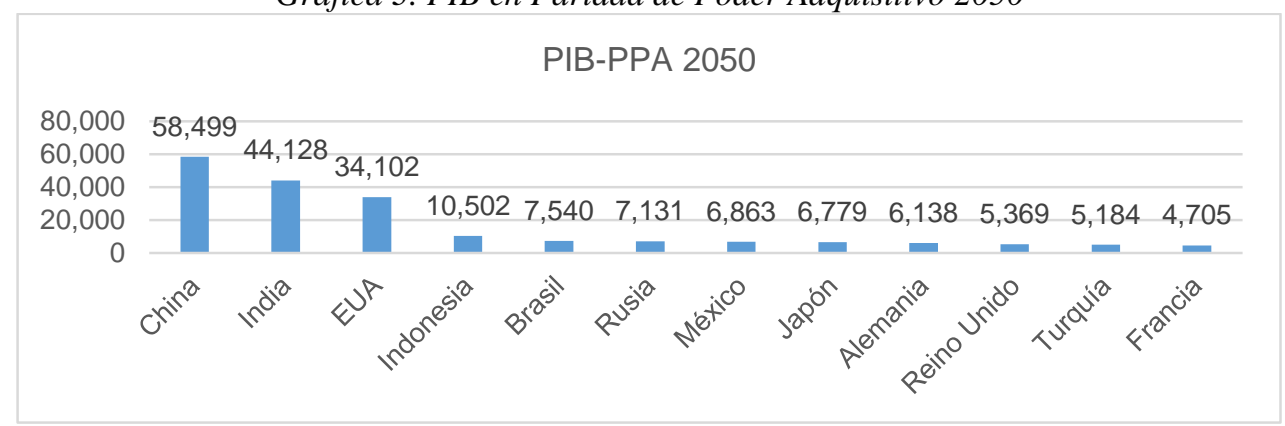

Fuente: PwC, 2021, Hacia el 2050: las economías emergentes tendrán el poder económico del mundo

Grafica 4. Proyección de crecimiento de México PIB - PPA 2016, 2030 y 2050 


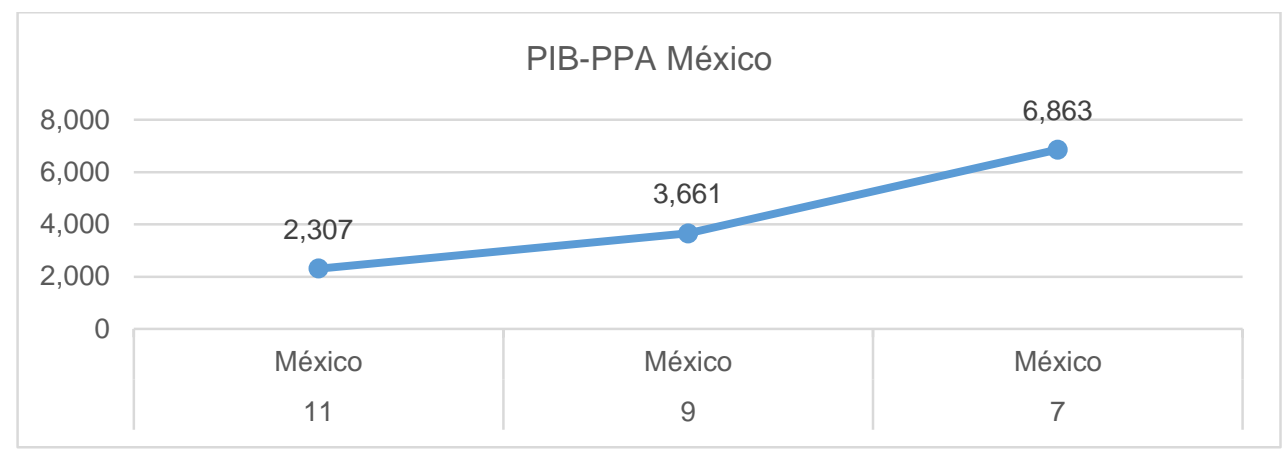

Fuente: PwC, 2021, Hacia el 2050: las economías emergentes tendrán el poder económico del mundo

Como podemos observar tanto en la Tabla 1. PIB en Paridad de Poder Adquisitivo (PPA), como en las gráficas 1, 2, 3 y 4, vemos que las proyecciones ponen a China por arriba de EUA como principal país en crecimiento de acuerdo con el PIB en Paridad de Poder Adquisitivo (PPA) en el año 2016, cabe mencionar que la posición que tiene México está por encima de países (incluso) del G7. Para el año 2030, se estima que ese orden del PIB-PPA puede cambiar poniendo a México, 2 puestos por arriba del año 2016. Mientras que para el año 2050, México escalonaría +2 puestos más, es decir, 4 puestos en total desde el año 2016 al 2050. Cabe insistir, siempre y cuando, se cumpla el promedio de crecimiento en la tasa anual del PIB de 2016 a 2050 que es de 5,012 puntos, es decir, durante los próximos 34 años. En términos de Ingresos Promedio en Latinoamérica, Argentina tendría un crecimiento promedio del 3\% anual, por arriba de Brasil y por debajo de Colombia y México.

Sin embargo, de acuerdo con el informe, el crecimiento global disminuirá a medida y relativamente cuando las poblaciones vayan envejeciendo y los países emergentes tomen posición las economías jóvenesmaduras, ya que para la década de 2020, caerá en un $2.7 \%$; 30`s, $2.5 \%$; y 40 `s $2.4 \%$.

Es menester lo siguiente, también de acuerdo con el informe, los grandes desafíos de los gobiernos, serán políticas de crecimiento sostenible a largo plazo (plan de desarrollo), es decir, para que dichas proyecciones se puedan realizar, las economías emergentes, deberán realizar inversiones sostenidas, uso de energías renovables, inversiones en educación, infraestructura y tecnología; ya que la necesidad de desarrollo instituciones políticas económicas, legales y sociales podrían generar incentivos sobre innovación, confianza en las finanzas, economías y estabilidad para la generación de nuevos negocios y confianza de inversión tras las continuas caídas de los precios de petróleo a nivel mundial desde 2014 a 2018.

Por ejemplo, los Estados Unidos de América, está trabajando para asegurar que aquel país a) logre una economía de energía limpia al 100\% y alcance emisiones netas CERO para el año 2050, solicitando a que el congreso incentive una ley que establezca un mecanismo de cumplimiento; una inversión económica para energías limpias, investigación e innovación para combatir el cambio climático; y el uso de innovaciones de energía limpia en comunidades más afectadas. Asimismo, b) convocar a países para combatir el cambio climático mediante el Acuerdo de París que habla sobre dicho problema, debiendo ser transparentes y aplicables, evitando falsedad en información, tratados y cumplimientos en políticas internacionales y comerciales como política exterior y de seguridad; c) enfocarse en las condiciones laborales de los trabajadores e impulsar de nueva cuenta una revolución industrial de energías limpias al cuidar las entidades económicas productoras de economías; y d) realizará una inversión circunstancial en energías limpias y justicia social, sin que hayan incentivos fiscales, con una inversión de $\$ 1.7$ a $\$ 5$ billones de dólares para los próximos 10 años.

Por otra parte, en una entrevista de CNN de Andrés Oppenheimer a Bill Gates, se tratan temas muy congruentes con el informe del PwC: se habla sobre el Acuerdo de París, en el que se convoca a que los 
países que conforman dicho acuerdo se unan y comprometan para reducir las emisiones de gases de efecto invernadero, sin embargo, no sería suficiente, ya que los países desarrollados y en desarrollo deberán realizar acciones de forma sustentable con el medio ambiente, pero hoy por hoy, es costoso; por lo que se deberán realizar innovaciones ramas comerciales e industriales como la biofarmacología, la bioalimentación, y biocombustibles. Por dichas causas, el cambio climático hará que las aguas de los mares inunden algunas tierras como en Miami y Nueva Orleans.

De igual forma, Gates habla de las Políticas Públicas en un sentido de desarrollar un plan real y generar interés para llegar a la meta que tiene el gobierno de EUA para llegar a cero emisiones de gases de efecto invernadero, para lo cual plantean un plan integral. También menciona que el sistema educativo de México es débil y que debería ser más importante que la inversión en petróleo, ya que una buena política educativa permitiría tener mexicanos informados, preparados y con un buen Ingreso Per cápita o Poder Adquisitivo adecuado, ya que el país no tiene como su principal recurso los energéticos, sino su propia mano de obra, la gente trabajadora, los empleados, los colaboradores, los asalariados.

No es suficiente, según Gates pronunciarse con el voto de la gente y hacer valer la voz del gobierno, sino más bien, es importante que el gobierno genere las bases que promuevan e incentiven una nueva cultura social, industrial y comercial acorde con el medio ambiente, en primera para cuidar los efectos de los gases invernaderos, cuidar el medio ambiente, y en segunda para tener un país con ventajas competitivas y comparativas y tratar de cumplir con las proyecciones de PwC Global, cumplir con los tratados del T-MEC, sobre todo porque EUA y Canadá, así como diversas asociaciones y cámaras comerciales, solicitan, requieren y reclaman energías sustentables, renovables, limpias; en las que no dependen solo de particulares, sino del portavoz mayor de un país.

Como bien sabemos, la industria automotriz es un buen indicador de las economías, y México hoy por hoy puede convertirse en una de las principales economías a futuro, no por volúmenes de hidrocarburos, sino más bien en temas de litio, ya que es un mineral considerado el nuevo petróleo, sin embargo, las malas decisiones de los gobiernos de las RIG's pudieran no enfocar correctamente su productividad y remanencia; ya que en el Estado de Sonora, existe un yacimiento muy rico en litio, conocido como el más grande del mundo, por lo que abre una brecha entre funcionarios políticos, inversionistas extranjeros y el discurso público.

Este mineral, al entrar en contacto con agua, se utiliza para fabricar cerámica, antidepresivos, productor en la industria nuclear y sobre todo, utilizado en baterías de telefonía móvil, computadoras, sistemas de almacenamiento eléctrica y baterías para autos... teniendo muy en cuenta éste último, ya que como se dijo anteriormente, la industria automotriz (indicador económico potencial) apuesta por este tipo de energías; del año 2014 al 2018, el precio de litio se disparó en un 150\%, es decir de \$6.69 dólares la tonelada a $\$ 17$ dólares respectivamente, por lo que la industria tecnológica para ser para México, su mayor oportunidad de crecimiento económico global permitiendo la oportunidad de abordar los lugares proyectados por PwC.

Tan solo, la empresa del magnate Elon Musk de la automotriz Tesla, produce diariamente 3 mil baterías con un aproximado de 13 kilogramos cada una, por lo que se requieren 24 mil toneladas al año para la producción automotriz de la marca, por lo que el T-MEC abre una puerta para la explotación de dicho mineral, ya que como uno de loso tratados, es que para dentro de tres años, se habrán de fabricar autos con un $75 \%$ de sus mecanismos y partes regionales libres de aranceles, por lo que se tendrá que desarrollar una cadena de suministro y distribución que involucra a los países del T-MEC.

\section{METODOLOGÍA}


Esta investigación es de manera cualitativa, ya que nos basamos en identificar las principales problemáticas de la investigación para poder buscar información en diversas fuentes que nos permitieran tener una literatura o marco de referencia (como las ya vistas en el Marco Teórico) para asemejar o proponer posibles modelos que permitan desarrollar una toma de decisión.

De acuerdo con las fuentes consultadas, realizamos el análisis de la información recabada sobre el panorama que presenta el día de hoy el país, así como sus necesidades y áreas de oportunidad para poder llevar a cabo un Plan de Desarrollo, sabiendo, que efectivamente, se debe realizar desde las altas autoridades federales, hasta el nivel de la sociedad para concientizar a la población en general de lo que se requiere para poder repuntar y trabajar con energías renovables; que si bien, los esfuerzos del gobierno federal, no han sido los más óptimos para alcanzar dichos objetivos, por el contrario, se han estancado al no mostrar interés sobre este tema, y por ende, la sociedad no cuenta con una cultura de desarrollo sustentable, entendiendo que la sociedad carece de cultura y valores que comprendan la importancia de este término al no tener una imagen representativa de lo que sucede, de lo que se plantea y de lo que se desea obtener, debido a que los valores y la cultura, según Burgoa, Herrera y Treviño (2013) son normas de conducta que rigen la forma de actuar de los individuos sobre lo que es bueno y malo considerados como fundamentos principales de las organizaciones en general, presentándose en las costumbres sociales y patrones de comportamiento incentivando el origen de relación cultural.

Por otra parte, visualizar los recursos con los que cuenta el país para ser generador de producciones ampliamente competitivas de acuerdo a la diversidad de los rubros mercantiles tanto de recursos como mercadológicos que se encuentran en los mercados, ya que deben satisfacer las necesidades de los consumidores (Herrera, Balderas \& Chapa, 2012), y en este sentido se tomaron en cuenta las variables de Producto Interno Bruto y Paridad de Poder Adquisitivo, (PIB y PPA respectivamente) obtenidas del PwC, 2021, las cuales nos muestran las proyecciones para los años 2030 y 2050, teniendo en cuenta que, las fuentes de energía derivadas del petróleo, ya no serán muy viables para el desarrollo de las economías, por lo que en este sentido, se pone como ejemplo la utilización del litio como probable precursor amigable con el medio ambiente y potencializador de manufactura de baterías.

De acuerdo a lo ya visto en líneas anteriores, se decidió realizar un modelo que abarca desde las estructuras gubernamentales, hasta los sectores económicos del país en el que se pretende que las instancias correspondientes establezcan Políticas Públicas Sustentables que aborden desde el campo, las industrias, la sociedad, los mercados y todo agente en general involucrado a la realización y ejecución de llevar a cabo una nueva cultura de Transformación Sostenible, por lo que se presenta el siguiente modelo como propuesta:

Tabla 2. Propuesta de Modelo: Energías Renovables, Sostenibles y Limpias de México

\begin{tabular}{|c|c|c|c|c|}
\hline & \multicolumn{4}{|c|}{ Sectores Económicos } \\
\hline & Primario & Secundario & Terciario & Cuaternario \\
\hline \multirow{2}{*}{$\begin{array}{l}\text { Generación de } \\
\text { Políticas } \\
\text { Públicas } \\
\text { sobre Energías } \\
\text { Renovables, } \\
\text { Sostenibles y } \\
\text { Limpias } \\
\text { (1) }\end{array}$} & $\begin{array}{l}\text { Agricultura, ganadería, } \\
\text { silvicultura y pesca }\end{array}$ & $\begin{array}{l}\text { Transformación, } \\
\text { minería y petróleo }\end{array}$ & $\begin{array}{c}\text { Turismo, comercio, } \\
\text { comunicaciones, } \\
\text { servicios y transportes }\end{array}$ & $\begin{array}{l}\text { Conocimiento, generación } \\
\text { e intercambio de } \\
\text { información, tecnología, } \\
\text { consultoría, educación, } \\
\text { investigación, desarrollo y } \\
\text { planificación financiera }\end{array}$ \\
\hline & $\begin{array}{c}\text { Inversión, } \\
\text { Transparencia, } \\
\text { Acuerdos, Políticas y } \\
\text { Normativas }\end{array}$ & \multicolumn{2}{|c|}{$\begin{array}{l}\text { Políticas laborales, educativas, energéticas, } \\
\text { económicas y sociales }\end{array}$} & $\begin{array}{l}\text { Inversión, Acuerdos } \\
\text { Mercantiles y Comerciales, } \\
\text { Políticas y Normativas }\end{array}$ \\
\hline
\end{tabular}


Estatutos para las materias primas

(2)
Ejecución y aplicación para la Innovación de Cultura Industrial, Comercial y Social (4)

Estatutos para acciones de $\mathrm{I}+\mathrm{D}+\mathrm{i}$

(3)

>> Concentración de Políticas Públicas de extremos hacia el centro de los Sectores Económicos

\section{$<<<$}

Fuente: Propia, Herrera, Treviño \& Cerecedo, 2021

\section{CONCLUSIONES:}

Como hemos visto, se ha presentado un buen panorama para México, así como para américa latina, pero especialmente para que lo proyectado por parte de $\mathrm{PwC}$, de empresarios de primer nivel como los mencionados Bill Gates y Elon Musk, e incluso por parte de algunas calificadoras de riesgo como Moody's, visualizan una previsión de crecimiento para México y países emergentes por perspectivas de expansión; no obstante, queda claro que por parte del gobierno debe formular y establecer lineamientos enfocados para ser un país competitivo, comparativo, eficiente, efectivo, innovador, tecnológico y moderno a través de la implementación de energías sustentables, renovables y limpias a fin de generar y proyecciones económicas y finanzas sanas, oportunidades de incrementar el poder adquisitivo de la sociedad, y ser patentes en la reducción de las emisiones de carbono, de los efectos de los gases invernaderos y el cambio climático.

De la misma manera, implementar los estatutos de Estado que converjan Políticas Públicas para las RIG's, las cuales incluyan normativas de inversión, transparencia, empleabilidad y laboral, educativa, energéticas, económicas, sociales y culturales, acuerdos mercantiles y comerciales; en donde la innovación del país puede recaer en sensibilizar, comunicar y difundir a la sociedad, a las industrias y comercios el enfatizar un cambio de conciencia y de oportunidad, y dar positivamente esa expectativa de que no se requieren de hidrocarburos, sino de inversiones en investigación y desarrollo para nuevas tecnologías limpias.

Tabla 3. Flujo de Modelo 


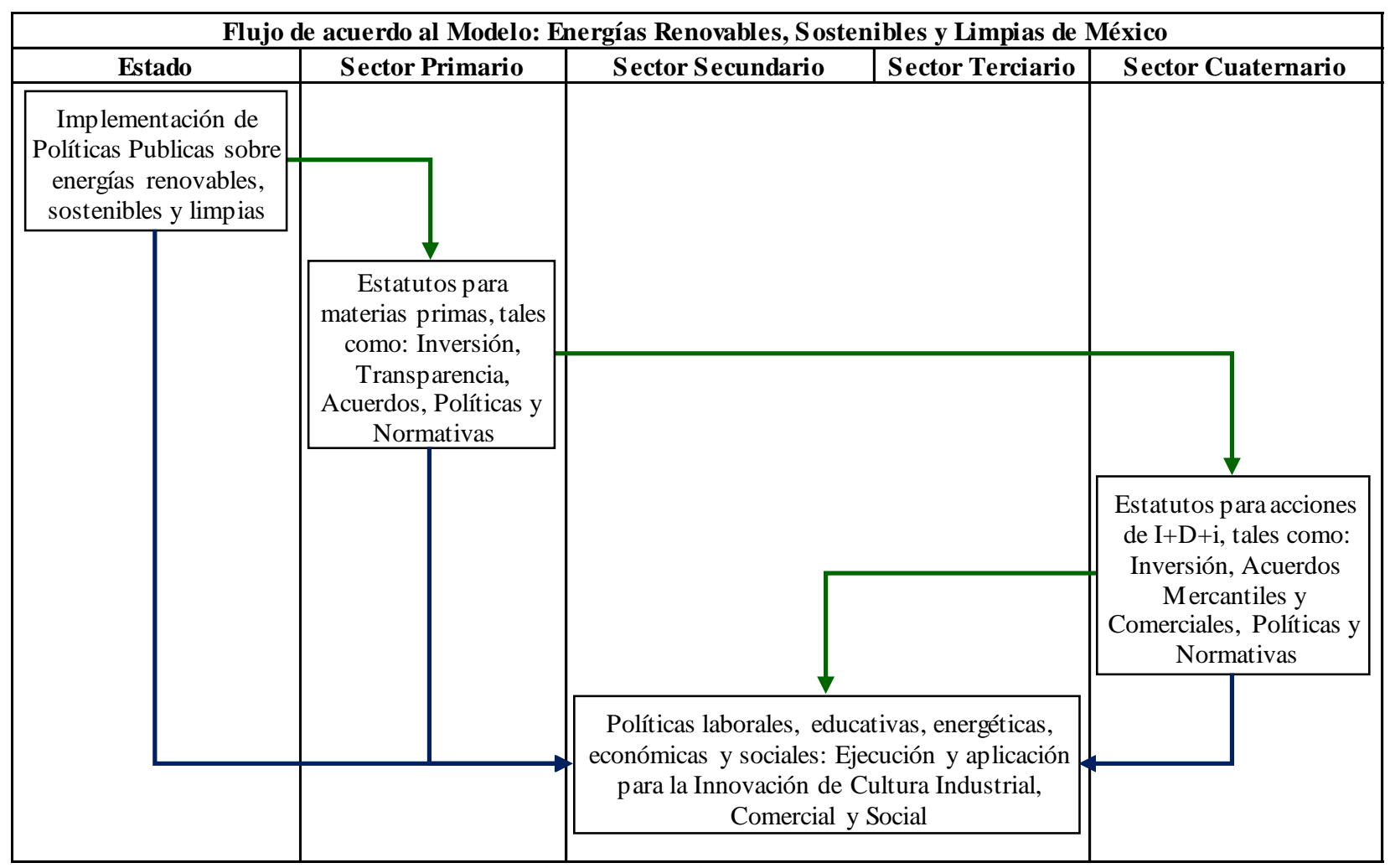

Fuente: Propia, Herrera, Treviño \& Cerecedo, 2021

\section{BIBLIOGRAFÍA}

Badii, M.; Castillo, J.; Guillén, A. \& Abreu, J. (abril de 2007). Sustentabilidad y política. Daena: International Journal of Good Conscience, 2(2), 274 - 285. Recuperado el 2021 de enero, de http://www.spentamexico.org/v2-n2/2(2)\%20274-285.pdf

Barcelata, H. (2012). Desarrollo industrial y dependencia económica en México. Alemania: Académica Española . Recuperado el enero de 2021, de https://www.uv.mx/personal/hbarcelata/files/2014/05/Desarrolloindustrialydependenciaecono micaenMexico.pdf

Barragán, A. (06 de septiembre de 2020). El País. Recuperado el enero de 2021, de El litio en México: entre los intereses privados y el discurso político: https://elpais.com/mexico/2020-09-06/el-litioen-mexico-entre-los-intereses-privados-y-el-discurso-politico.html

Burgoa, T., Herrera, E. \& Treviño, J. (2013). Estudio sobre la administración de empresas familiares en México: Principales problemas y retos que enfrentan. Daena: International Journal of Good Conscience, 2(8), 1-22.

Carrillo Fernández, O. F. (Enero-Junio de 2012). Tendencias mundiales de producción y consumo de energía y reducción de emisiones de CO2. Legado, $7(11), 61-78$. Recuperado el 12 de junio de 2020, de https://legadodearquitecturaydiseno.uaemex.mx/article/view/14035/10736 
Forbes, S. (24 de febrero de 2021). Forbes México. Recuperado el febrero de 2021, de Moody's sube proyección de crecimiento de México junto a países emergentes:

https://www.forbes.com.mx/economia-moodys-proyeccion-crecimiento-mexico-grupo-paisesemergentes/

Gurría, A. (03 de octubre de 2011). OCDE.org. Recuperado el diciembre de 2020, de El G20: Países Emergentes, Gobernanza Global y Desarrollo:

https://www.oecd.org/fr/apropos/secretairegeneral/elg20paisesemergentesgobernanzaglobaly desarrollo.htm

Herrera, E., Balderas, J. \& Chapa, R. (2012). Análisis sobre el perfil de directivos empresariales en México: caso de estudio. Innovaciones de Negogicios, 17(9), 109-128.

Hodson de Jaramillo, E. (julio - septiembre de 2018). Bioeconomía: el futuro sostenible. Revista académica Colombiana de ciencias exactas, físicas y naturales, 164(42), 188 - 201. Recuperado el enero de 2021, de http://www.scielo.org.co/pdf/racefn/v42n164/0370-3908-racefn-42-16400188.pdf

Jacobs, M. (1991). The Green Economy. London.: Pluto Press.

joebiden.com. (2021). Batalla por el alma de la nación. Recuperado el febrero de 2021, de https://joebiden.com/es/el-plan-biden-para-una-revolucion-de-energia-limpia-y-justiciaambiental/

Naciones Unidas, N. (25 de diciembre de 2015). Objetivos de desarrollo sostenible. Recuperado el febrero de 2021, de La Asamblea General adopta la Agenda 2030 para el Desarrollo Sostenible: https://www.un.org/sustainabledevelopment/es/2015/09/la-asamblea-general-adopta-laagenda-2030-para-el-desarrollo-sostenible/

Oppenheimer, A. (23 de febrero de 2021). CNN Español. Recuperado el febrero de 2021, de Bill Gates: México puede tener un futuro muy brillante sin el petróleo porque su principal recurso es su gente: https://cnnespanol.cnn.com/2021/02/23/bill-gates-entrevista-andres-oppenheimer/

Oropeza, A. (2013). México en el desarrollo de la Revolución Industrial: evaluaciones y perspectivas. (I. p. económico, Ed.) Biblioteca jurídica virtual del instituto de investigaciones jurídicas, 199 - 236.

Recuperado el enero de 2021, de https://archivos.juridicas.unam.mx/www/bjv/libros/7/3371/10.pdf

PwC. (2021). Hacia el 2050: las economías emergentes tendrán el poder económico del mundo.

Recuperado el febrero de 2021, de https://www.pwc.com.ar/es/prensa/hacia-2050-economiasemergentes-tendran-poder-economico-del-mundo.html

Ramírez, A.; Sánchez, J.; García, A. (julio - diciembre de 2004). El desarrollo sustentable: Interpretación y Análisis. Revista del Centro de Investigación, 55-59. Recuperado el enero de 2021, de https://www.redalyc.org/pdf/342/34202107.pdf

Rodríguez, I. (19 de junio de 2020). Expansión. Recuperado el enero de 2021, de Baterías de litio: la nueva oportunidad que abre el T-MEC para Norteamérica: 
https://expansion.mx/empresas/2020/06/19/baterias-de-litio-la-nueva-oportunidad-que-abreel-t-mec-para-norteamerica

Seefoó Luján, J. L. (2014). Hacia la sustentabilidad ambiental de la producción de energía en México. Relaciones. Estudios de historia y sociedad, 35(139), 343-350. Hacia la sustentabilidad ambiental de la producción de energía en México. Relaciones. Estudios de historia y sociedad, 35(139), $343-$ 350. Recuperado el 16 de junio de 2021, de http://www.scielo.org.mx/scielo.php?script=sci_arttext\&pid=S0185$39292014000300015 \& \operatorname{lng}=e s \&$ tlng=es.

Times, F. (2021). The Financial Times. Recuperado el febrero de 2021, de Tesla en disputa por suministro de litio: https://www.ft.com/content/4a924a64-99df-11e5-987b-d6cdef1b205c

\footnotetext{
*Acerca de los Autores

Emmanuel Herrera es Ingeniero Industrial Administrador, posee una Maestría en Administración Industrial y de Negocios, labora en el Instituto de Educación Superior Rosario Castellanos, de la Secretaría de Educación, Ciencia, Tecnología e Innovación de la Ciudad de México. Correo electrónico: ing.e.herrera@hotmail.com.
}

Jorge Treviño es Maestro en Administración Pública y cuenta con un Doctorado en Educación, labora como coordinador de licenciatura y docente en la Facultad de Contaduría Pública y Administración de la Universidad Autónoma de Nuevo León. Correo electrónico: jgtrevinom@ yahoo.com.mx

Eduardo Cerecedo es Contador Público, tiene una maestría en Educación y Docencia, labora en el Instituto de Educación Superior Rosario Castellanos, de la Secretaría de Educación, Ciencia, Tecnología e Innovación de la Ciudad de México. Correo electrónico: eduardocc_contabilidad@ hotmail.com

Reconocimientos: Los autores desean expresar sus agradecimientos a las siguientes personas que contribuyeron con su amable apoyo en las sugerencias de mejora del contenido de esta publicación: Dr. Luis Alberto Villarreal Villarreal, FACPYA, UANL; Dr. José Nicolás Barragán, FACPYA, UANL; Dr. Luis Chávez Guzmán, FIME, UANL; Lic. Bertha Sánchez, Secretaría de Relaciones Exteriores; Dra. Nahiely Flores, IRC; C. Natalia Velázquez; C. Leonardo Herrera.

Aknowledgement: The authors wish to express their gratitude to the following people who contributed with their kind support in the suggestions for improving the content of this publication: Dr. Luis Alberto 
Villarreal Villarreal, FACPYA, UANL; Dr. José Nicolás Barragán, FACPYA, UANL; Dr. Luis Chávez Guzmán, FIME, UANL; Dra. Nahiely Flores, IRC; C. Natalia Velázquez; C. Leonardo Herrera. 